\title{
Macroporous MgO monoliths prepared by sol-gel process with phase
}

\section{separation}

\author{
Meiting Li ${ }^{a}$, Ningning Zhou ${ }^{b}$, Xudong Luo ${ }^{a, c, *}$, Guodong Zhang ${ }^{a}$, Zhipeng Xie ${ }^{c}$, Linchong Xu ${ }^{a}$, \\ Pengcheng Liu ${ }^{a}$ \\ ${ }^{\text {a }}$ School of High Temperature Materials and Magnesium Resource Engineering, Liaoning \\ University of Science and Technology, Anshan 114051, P. R. China \\ ${ }^{\mathrm{b}}$ Liaoning Petrocchemical Vocational and Technology, Jinzhou 121001, P. R. China \\ ${ }^{\mathrm{c}}$ Key Laboratory of New Ceramics and Fine Processing, School of Materials Science and \\ Engineering, Tsinghua University, Beijing 100084, P. R. China
}

\begin{abstract}
Macroporous $\mathrm{MgO}$ monoliths were prepared with magnesium chloride $\left(\mathrm{MgCl}_{2} \cdot 6 \mathrm{H}_{2} \mathrm{O}\right)$ as a precursor, poly(ethylene oxide) (PEO) as a phase-separation inducer, and propylene oxide (PO) as a gelation agent via sol-gel processing. Based on the results of differential thermal analysis (TG-DTA), Fourier transform infrared (FT-IR) spectroscopy, X-ray diffractometry (XRD), and scanning electron microcopy (SEM), the effects of PEO addition on sinterability and microstructure of the monoliths were discussed. It was found that the amount of PEO should be within a certain range, within which the gel skeletons were gradually strengthened, three-dimensional interconnective macroporous structures were resulted and the porosity of the sintered specimens obviously increased with increasing PEO addition. Furthermore, the surfaces of $\mathrm{MgO}$ particles were coated with some cylindrical fibres ranged from $800 \mathrm{~nm}$ to $2 \mu \mathrm{m}$ in
\end{abstract}

\footnotetext{
* Corresponding author: Xudong Luo (1980- ), male, doctor, associate professor. E-mail:luoxudongs@aliyun.com

Biography: Meiting Li (1990- ), female, postgraduate. E-mail:1191028917@qq.com
} 
diameter, and the quantity of the fibres is proportional to the PEO addition.

Keywords: sol-gel processing, macroporous $\mathrm{MgO}$ monoliths, $\mathrm{PEO}$ addition, $\mathrm{MgO}$ cylindrical fibre.

\section{Introduction}

Porous materials have large specific surface areas, high porosity, permselectivity for gas and liquid media ${ }^{[1,2]}$. The materials are thus being considered for promising applications as filters, column pickings for chromatography, sensors, water cleaners, heat insulators, catalyst supports, and ion exchangers ${ }^{[3,4]}$. Porous magnesia $(\mathrm{MgO})$ stands out in these areas owing to its high refractoriness, low thermal conductivity, high thermal insulation, and so forth. Currently, the preparation of porous materials is generally achieved via sol-gel processing ${ }^{[5]}$, freeze-drying ${ }^{[6]}$, organic foaming ${ }^{[7]}$, and with a method involving pore-forming agent ${ }^{[8]}$. As a type of wet-chemical techniques for high purity monoliths, sol-gel processing accompanied by phase separation is a novel approach to fabricate porous materials of high specific surface areas, interconnective macropores, and uniform pore sizes. Therefore, it has become a hot research topic worldwide ${ }^{[9]}$.

In the sol-gel process, a metal salt or an inorganic salt, used as precursor, is mixed with an aqueous organic solvent to form a homogeneous solution, which produces a hydrophobic-hydrophilic repulsive interaction between the solute and solvent, and thus a synthetic gel is formed. Meanwhile, adding an organic polymer into the solution may induce phase separation to produce xerogels by aging and drying ${ }^{[10]}$. Gash et al. ${ }^{[1]]}$ first investigated the sol-gel technique using aqueous solution of metal salts. In their work, the strong nucleophilic base of 
epoxide-mediated captured the dissociated $\mathrm{H}^{+}$to initiate an irreversible ring-opening reaction, which rapidly raised the solution $\mathrm{pH}$ to promote the hydrolysis and polymerization of hydrate metals ions. Nakanishi et al. ${ }^{[12]}$ systematically summarized the mechanisms of phase separation during sol-gel processing and further synthesized a series of porous monoliths such as $\mathrm{SiO}_{2}{ }^{[13]}$, $\mathrm{TiO}_{2}{ }^{[14]}, \mathrm{ZrO}_{2}{ }^{[15]}$, and mullite, ${ }^{[16]}$ through theoretical analysis of porous materials formation via sol-gel has yet to be fully matured. To be the best of our knowledge, sol-gel fabrication of porous magnesia monoliths with phase separation has seldom been reported. In the present work, porous $\mathrm{MgO}$ monoliths were prepared via a sol-gel process accompanied by phase separation, using magnesium chloride $\left(\mathrm{MgCl}_{2} \cdot 6 \mathrm{H}_{2} \mathrm{O}\right)$ as a precursor, poly (ethylene oxide) (hereafter referred to as PEO) as a phase separation inducer, and propylene oxide (hereafter referred to as PO for convenience) as a gelation agent. The effects of PEO addition on the sinterability and microstructure of the resultant macroporous $\mathrm{MgO}$ monoliths were elucidated.

\section{Experimental work}

\subsection{Raw materials}

Magnesium chloride $\left(\mathrm{MgCl}_{2} \cdot 6 \mathrm{H}_{2} \mathrm{O}, 98 \%\right.$ pure, Sinopharm Chemical Reagent Co., Ltd) was used as a precursor, poly (ethylene oxide, PEO, $M_{v}=10^{5}$, 99\% pure, Sinopharm Chemical Reagent Co., Ltd) was used as a phase-separation inducer, propylene oxide (PO, 99\% pure, Sinopharm Chemical Reagent Co., Ltd) was used as a gelation agent, and distilled water, tetraethoxysilane (TEOS, Sinopharm Chemical Reagent Co., Ltd), and ethanol (EtOH, analytical grade, Sinopharm Chemical Reagent Co., Ltd) were used as solvents. 


\subsection{Preparation}

Prescribed amounts of $\mathrm{MgCl}_{2} \cdot 6 \mathrm{H}_{2} \mathrm{O}$ and PEO were dissolved in a mixture of $\mathrm{H}_{2} \mathrm{O}$ and EtOH to form a transparent homogenous solution after continuous stirring for $2 \mathrm{~h}$ at room temperature. A certain amount of PO was then dripped into the solution while stirring for $1 \mathrm{~min}$. The resultant homogeneous solution was sealed and transferred into an oven kept at $80{ }^{\circ} \mathrm{C}$ to start the sol-gel transition and aging processes. Solvent exchange was carried out in the stock solution (the solution before doping PO) under ambient conditions by soaking for $24 \mathrm{~h}$. Firstly, EtOH replacement was performed twice, each time for $4 \mathrm{~h}$, to remove the water and other impurities in the original system. Afterward, the wet gel was immersed in an ethanol solution of TEOS for $24 \mathrm{~h}$. After gelation, the residual TEOS was removed by EtOH washing for two times, and the wet $\mathrm{MgO}$ gel was oven-dried at $40{ }^{\circ} \mathrm{C}$ for 3 days. Some of the resultant xerogels was finally heat-treated at $1100{ }^{\circ} \mathrm{C}$ for $2 \mathrm{~h}$. The specific experimental parameters are shown in Table 1.

\subsection{Characterisation}

Thermal behaviour of the specimens was investigated using a LabsysEvo STA equipment in the temperature range of $25-1450{ }^{\circ} \mathrm{C}$, with a heating rate of $5 \% \mathrm{~min}$. The functional groups built in the product were analyzed by Fourier transform infrared spectroscopy (FT-IR, Affinity-1, Shimadzu Corp., Japan). The porosity and bulk density of specimens after sintering were measured according to the Archimedes method using apparent porosity and bulk density tester (XQK-04, China). Phase structure of the products were analyzed via X-ray diffractometry (XRD, $\left.0.02^{\circ}, 0^{\circ} \leq 2 \theta \leq 90^{\circ}\right)$ using $\mathrm{Cu} \mathrm{K} \alpha$ radiation $(40 \mathrm{kV}, 100 \mathrm{~mA})$ and a scanning speed of $4^{\circ} \cdot \mathrm{min}^{-1}$. 
Microstructure of the dried gel was observed using a Zeiss $\Sigma$ IGMA field emission scanning electron microscope (Zeiss, Germany).

\section{Results and discussion}

\section{1 phase composition and microstructure of the porous magnesia materials}

Fig. 1 shows XRD pattern of Specimen 3 after sintering, where it is seen that periclase crystallized as the dominant phase. The analysis suggested that when PO was added to the precursor, the gelation and phase separation proceeded spontaneously under certain condition to produce a wet gel after $20 \mathrm{~min}$. The strong nucleophilic base of PO, as a gelation agent, could shorten the gelation time and capture the dissociated $\mathrm{H}^{+}$and induce protonation. Meanwhile, PO coupled with free $\mathrm{Cl}^{-}$, followed by an irreversible ring-opening reaction (see Eq. 1 and Eq. 2) ${ }^{[17]}$. Simultaneously, the resultant $\mathrm{H}^{+}$and $\mathrm{Cl}^{-}$would rapidly stimulate the hydrolysis of subsequently dropped TEOS which could improve the strength of the gel skeleton and avoid the volumetric shrinkage during drying ${ }^{[15]}$. The presence of $\mathrm{PO}$ caused an increase in solution $\mathrm{pH}$, and the gel for $\mathrm{MgO}$ was resulted via hydrolysis and polymerisation the hydrated $\mathrm{Mg}^{2+}$ ions. The residual organics were evaporated and decomposed upon high-temperature calcination, and porous magnesia was thus crystallized as the major phase.

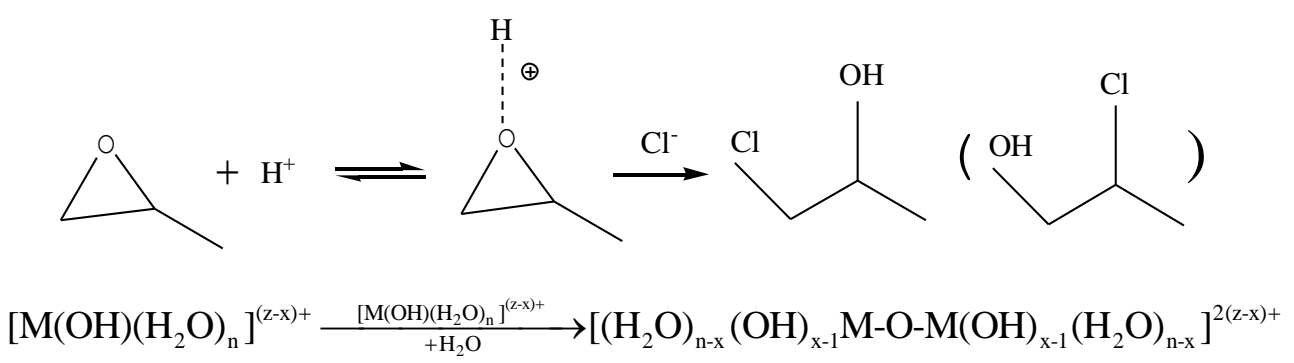

SEM images (magnified $2000 \times$ ) of the $\mathrm{MgO}$ xerogels are shown in Fig. 2. It is seen that the 
content of PEO determined the trend of phase separation and the morphology of the product thereof. The relationship between the trend of phase separation and the overall thermodynamics of the process can be described by the Flory-Huggins formulation (3) ${ }^{[18]}$ :

$$
\Delta \mathrm{G}=\mathrm{RT} \quad\left(\frac{\phi_{1} \ln \phi_{1}}{\mathrm{P}_{1}}+\frac{\phi_{2} \ln \phi_{2}}{\mathrm{P}_{2}}+\chi_{12} \phi_{1} \phi_{2}\right)
$$

where $\Phi_{1}$ and $\Phi_{2}$ are the volume fractions of two components in the system, $P_{1}$ and $P_{2}$ are the degrees of polymerisation, $\chi_{12}$ is the interaction parameter, and the former two terms and the last term in parentheses represent the entropic and the enthalpic contributions, respectively. With decreasing absolute value of entropy change, the free energy and the enthalpy change of the system will increase, and when $\Delta G$ changes from negative to positive, phase separation transformation will be inevitable. In the $\mathrm{MgO}$ sol-gel system, the ether-linkage of PEO preferentially adsorbs on the surface of $\mathrm{MgO}$ via hydrogen bonding due to the hydrophobic-hydrophilic repulsive interactions with this mixed solvent. Simultaneously, phase separation proceeded spontaneously, and an interconnective macroporous structure was therefore resulted as observed. It is remarkable that the phase separation process is realised as long as the PEO content is within a certain range. The growth of gel skeleton is attributed to increasing PEO addition, and a three-dimensionally interconnective macroporous structure was thereby accomplished. Phase separation did not occur with insufficient contents of PEO. With excessive PEO, on the contrary, the phase separation process was drastic. In this case, the gel skeleton was destroyed to reduce the surface area, which accelerated the agglomerations of $\mathrm{MgO}$ particles. Figs. 2(a)-(d) exhibit that the network skeletons were strengthened and the macroporous structure become clearer with increasing PEO addition. The PEO content was deficient in Fig. 2(a), and thus phase separation is not obvious. The PEO content was excessive in Fig. 2(e), and hence the 
particles of $\mathrm{MgO}$ xerogels began to aggregate. It is indicated that the strong interaction between polymers and solvents gave raise to destroyed macroporous structure. A comparation with Fig. 2(a) and Fig. 2(b) suggested that the porosity increased apparently due to the drastic phase separation effect, the pore size increased from $500 \mathrm{~nm}$ to $2 \mu \mathrm{m}$. Furthermore, porosimetry analysis found properly increasing PEO addition led to $\mathrm{MgO}$ xerogels having uniform pore sizes of 2-3 $\mu \mathrm{m}$ in diameter. It can thus be said that with the technique employed in this work, porous materials have not only large porosity, but also smaller pore sizes can be prepared. It is known that smaller pores help to improve structural strength of the porous material.

Introducing PEO can significantly influence the macroporous structure and crystalline morphology of $\mathrm{MgO}$ xerogels. Fig. 3 shows SEM images (magnified $5000 \times$ ) of the $\mathrm{MgO}$ xerogels, with Figs 3(a) to (e) for PEO contents ranging from $2.1 \mathrm{~g}$ to $2.5 \mathrm{~g}$, respectively. As confirmed by SEM observation, a large amount of $\mathrm{MgO}$ cylindrical fibres grew on the surfaces of $\mathrm{MgO}$ particles. Spherical and plate-like $\mathrm{MgO}$ particles began to grow preferentially in a certain axial direction with some having a laminar structure, and some having a more thickset structure. The cylindrical fibres increased in number and length in a particular orientation, and the fibre diameters was observed to increase from $800 \mathrm{~nm}$ to $2 \mu \mathrm{m}$ with increasing PEO addition (Figs 3(b)-(d)). The PEO content was excessive in Fig. 3(e), and the particles of $\mathrm{MgO}$ xerogel began to aggregate. $\mathrm{MgO}$ fibre-coating layers existed on the surfaces of $\mathrm{MgO}$ particles, but the growth of cylindrical fibres was restrained. It was illustrated that the $\mathrm{MgO}$ fibres grew in a preferential orientation and the addition of PEO promoted their growth. Too much PEO, however, inhibited fibre growth. The $\mathrm{MgO}$ fibres bridged the gel skeleton, and thus the strength of the monoliths increased. In addition, high magnification observation (Fig. 3(f), magnified 20,000 $\times$ from Fig. 3(c)) indicated that the 
cylindrical micro-fibres have tubular substructures. Considered from the principles of fibre toughening, the observed fibres may improve the toughness and thermal shock resistance, and thus the working life, of the $\mathrm{MgO}$ xerogel. Moreover, the increased porosity may reduce the thermal conductivity of the xerogel and improve the thermal insulation performance thereof.

On the basis of the enlarged pictures (magnified 10,000 $\times$ ) of macroporous morphology (the upper right corner of Fig. 3(a), Fig. 3(c) and Fig. 3(e)), as the MgO fibre content increased, the sizes of macropores increased gradually. Moreover, the increase in PEO content increased the pore volume. The interconnection between entangled fibres formed a three-dimensional network. The decreasing content of spherical and plate-like $\mathrm{MgO}$ particles and the increasing number of pores were expected to improve the thermal insulation performance of the xerogel. Besides, when the content of PEO was $2.50 \mathrm{~g}, \mathrm{MgO}$ cylindrical fibres became finer, and the internal solid skeleton collapsed or dissociated basically, $\mathrm{MgO}$ particles began to aggregate with the impact of gravity.

\subsection{Thermal analysis of the macroporous monoliths}

Fig. 4 displays TG/DTA curve of Specimen 3, where one endothermic peak and two exothermic peaks were found on the DTA curve. The endotherm near $136{ }^{\circ} \mathrm{C}$ is attributed to the removal of residual organic solvent in the gel. The organic species evaporate and tend to absorb water at elevated temperature. A small exothermic peak near $325{ }^{\circ} \mathrm{C}$ is related to the pyrolytic decomposition of PEO or decomposition of the product generated by the ring-opening reaction ${ }^{[19]}$. The exotherm near $468{ }^{\circ} \mathrm{C}$ may be ascribed to the pyrolytic decomposition of other organic species and/or the crystallization of $\mathrm{MgO}$. The $55.34 \%$ weight loss observed from the TG curve clarified the role of PEO as a phase-separation inducer which was preferentially distributed on the 
$\mathrm{MgO}$ gel skeleton.

The FT-IR spectra of Specimen 3 after drying and sintering at $1100{ }^{\circ} \mathrm{C}$ are shown in Fig. 5 . The two absorption bonds near 440 and $870 \mathrm{~cm}^{-1}$ arise from the bending vibration of $\mathrm{Mg}-\mathrm{O}$ bond, which illustrated the existence of Mg-O-Mg linkages in the gel stage. The two broad bands at 1643 and $3420 \mathrm{~cm}^{-1}$ are assigned to the stretching and bending vibration of the $\mathrm{O}-\mathrm{H}$ bond in $\mathrm{H}_{2} \mathrm{O}$. The weak absorption peak near $2922 \mathrm{~cm}^{-1}$ can be attributed to the C-H symmetric and asymmetric stretching vibrations characteristic of $\mathrm{PEO}^{[20]}$. This further demonstrated that $\mathrm{PEO}$ adsorbed on the surface of $\mathrm{MgO}$ and captured the dissociated $\mathrm{H}^{+}$to induce irreversible ring-opening reaction. In addition, the vibration of $\mathrm{Mg}$-O bond got strengthened after sintering at $1100{ }^{\circ} \mathrm{C}$, when compared with that of the dried gel. The above FT-IR analyses are in accordance with the results of TG/DTA.

\subsection{Macro-property analysis of the sintered samples}

$\mathrm{PEO}$, as the phase-separation inducer of this $\mathrm{MgO}$ gel system, affected the porosity and bulk density of the $\mathrm{MgO}$ xerogel. The bulk density and porosity of the sintered specimens with different initial PEO contents are shown in Fig. 6. It was found that the bulk density first increased and then decreased with increasing PEO content, while the porosity changes in an opposite way. This suggested that the phase separation process was evident when the PEO content was within a certain range. That is the increasing phase separation rate with increasing PEO addition promoted the formation of an inner macroporous structure in the monoliths, and thus the porosity increased. When exceeding the range, phase separation was aggravated and, as a result, the gel skeleton was destroyed. The decreased porosity of the sintered specimens may detrimentally affect the 
performance of the porous materials.

\section{Conclusion}

Porous $\mathrm{MgO}$ monoliths were prepared using a sol-gel process with phase separation, and the effects of PEO addition on sinterability and microstructure of the $\mathrm{MgO}$ monoliths were studied. It was shown that only when the amount of PEO was within a certain range, the gel skeletons grew and the porosity of the sintered specimens increased along with increasing PEO addition. When the PEO content was excessive, the gel skeleton collapsed due to drastic phase separation, and the aggregated $\mathrm{MgO}$ particles reduced the porosity of the $\mathrm{MgO}$ porous monoliths. Meanwhile, cylindrical $\mathrm{MgO}$-fibre coating layers were formed on the surfaces of $\mathrm{MgO}$ particles. Increasing PEO content led to more fibres, whose diameters increased from $800 \mathrm{~nm}$ to $2 \mu \mathrm{m}$. Besides, the sintered specimens were mostly periclase, and the purity of the $\mathrm{MgO}$ porous material was thereby improved.

\section{Acknowledgements}

This work was financially supported by National Science Foundation of China. (No.51402143)

\section{References}

[1] S.F. Corbin, P. S. Apté. Engineered Porosity via Tape Casting, Lamination and the Percolation of Pyrolyzable, Journal of the American Ceramic Society 82(7) (1999) 693-704. 
[2] W.H. Zi, J.S. Sun, M.H. Huang, et al. Research process of sol-gel preparing porous ceramic, China Ceramics 39(4) (2003) 14-18.

[3] H. Abe, H. Seki, A. Fukunaga, et al. Preparation of bimodal porous mullite ceramics, Journal of Materials Science29(5) (1994) 1222-1226.

[4] J.H. She, T. Ohji. Fabrication and characterization of highly porous mullite ceramics, Materials Chemistry and Physics 80(3) (2003) 610-614.

[5] K. Nakanishi, N. Tanaka. Sol-gel with phase separation. Hierarchically porous materials optimized for high-performance liquid chromatography separations, Accounts of Chemical Research 40 (2007) 863-873.

[6]H.J. Yoon, U.C. Kim, J.H. Kim, et al. Macroporous alumina ceramics with aligned microporous walls by unidirectionally freezing foamed aqueous ceramic suspensions, Journal of the American Ceramic Society 93(6) (2010) 1580-1582.

[7]X.J. Mao, S.W. Wang, S.J. Shimai. Porous Ceramics with Tri-modal Pores Prepared by Foaming and Starch Consolidation, Ceramics International 134(1) (2008) 107-112.

[8] K. Okada, M. Shimizu, T. Isobe, et al. Characteristics of microbubbles generated by porous mullite ceramics prepared by an extrusion method using organic fibers as the pore former, Journal of the European Ceramic Society 30(6) (2010) 1245-1251.

[9] K. Nakanishi. Pore structure control of silica gels based on phase separation, Journal of Porous Materials 42(2) (1997) 67-112.

[10] N. Kazuki. Functional porous materials via sol-gel with phase separation, Journal of the Ceramic Society of Japan 115(1339) (2007) 169-175.

[11] A.E.Gash, T.M.Tillotson, J.H. Satcher, et al. Strong akaganeite aerogel monoliths using epoxides: synthesis and characterization, Chemistry of Materials 15 (2003) 3268-3275. 
[12] K. Nakanishi. Pore structure control of silica gels based on phase separation, Journal of Porous Materials 4 (1997) 67-112.

[13] K. Nakanishi, H. Soga. Phase separation in silica sol-gel system containing poly(ethylene oxide). II, Effects of molecular weight and temperature, Bulletin of the Chemical Society of Japan 70 (1197) 587-592.

[14] W.Y. Li, X.Z. Guo, Y. Zhu, et al. Sol-gel synthesis of macroporous $\mathrm{TiO}_{2}$ from ionic precursors via phase separation route, Journal of Sol-Gel Science and Technology 67(3) (2013) 639-645.

[15] X.Z. Guo, L. Lü, H. Yang. Preparation of macroporous zirconia monoliths via sol-gel process accompanied by phase separation, Journal of the Chinese Ceramic Society 42(1) (2014) 6-10.

[16] X.Z. Guo, W.Y. Li, K. Nakanishi, et al. Preparation of mullite monoliths with well-defined macropores and mesostructured skeletons via the sol-gel process accompanied by phase separation, Journal of the European Ceramic Society 33(10) (2013) 1967-1974.

[17] Y. Kido, K. Nakanishi, A. Miyasaka, et al. Synthesis of monolithic hierarchically porous iron-based xerogels from iron(III) salts via an epoxide-mediated sol-gel process 2(11) (2012) 2071-2077.

[18] J. Konishi, K. Fujita, S. Oiwa, et al. Crystalline $\mathrm{ZrO}_{2}$ monoliths with well-defined macropores and mesostructured skeletons prepared by combining the alkoxy-derived sol-gel process accompanied by phase separation and the solvothermal process, Chemistry of materials 20(6) (2008) 2165-2173.

[19] X.Z. Guo, W.Y. Li, Y. Zhu, et al. Macroporous $\mathrm{SiO}_{2}$ monoliths prepared via sol-gel process accompanied by phase separation, Acta Physico-Chimica Sinica 29(3) ( 2013) 646-652.

[20] Z.M. Shi, F. Pan, D.Y. Liu, et al. Effect of $\mathrm{Ce}^{4+}$-modified amorphous $\mathrm{SiO}_{2}$ phase transformation towards $\alpha$-cordierite, Materials Letters 57(2) (2002) 409-413. 


\section{FIGURE CAPTIONS}

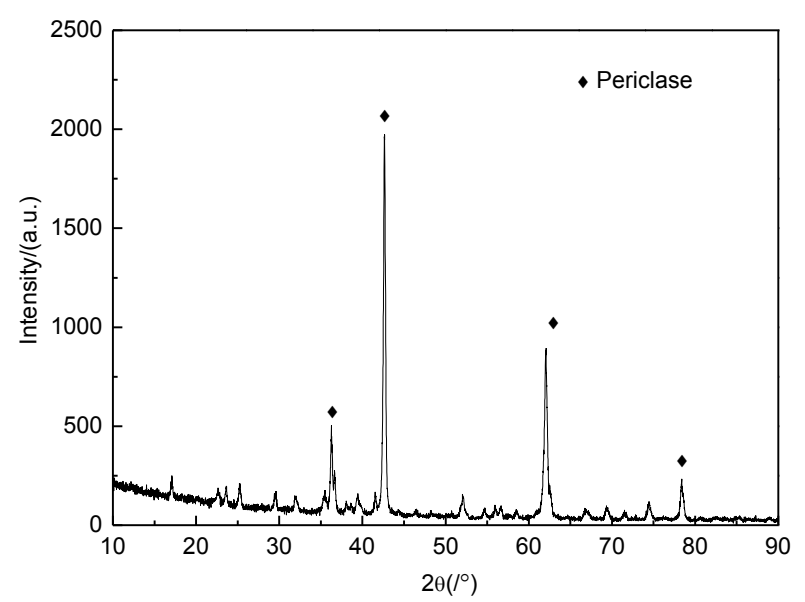

Fig. 1 XRD pattern of Specimen 3 after sintering
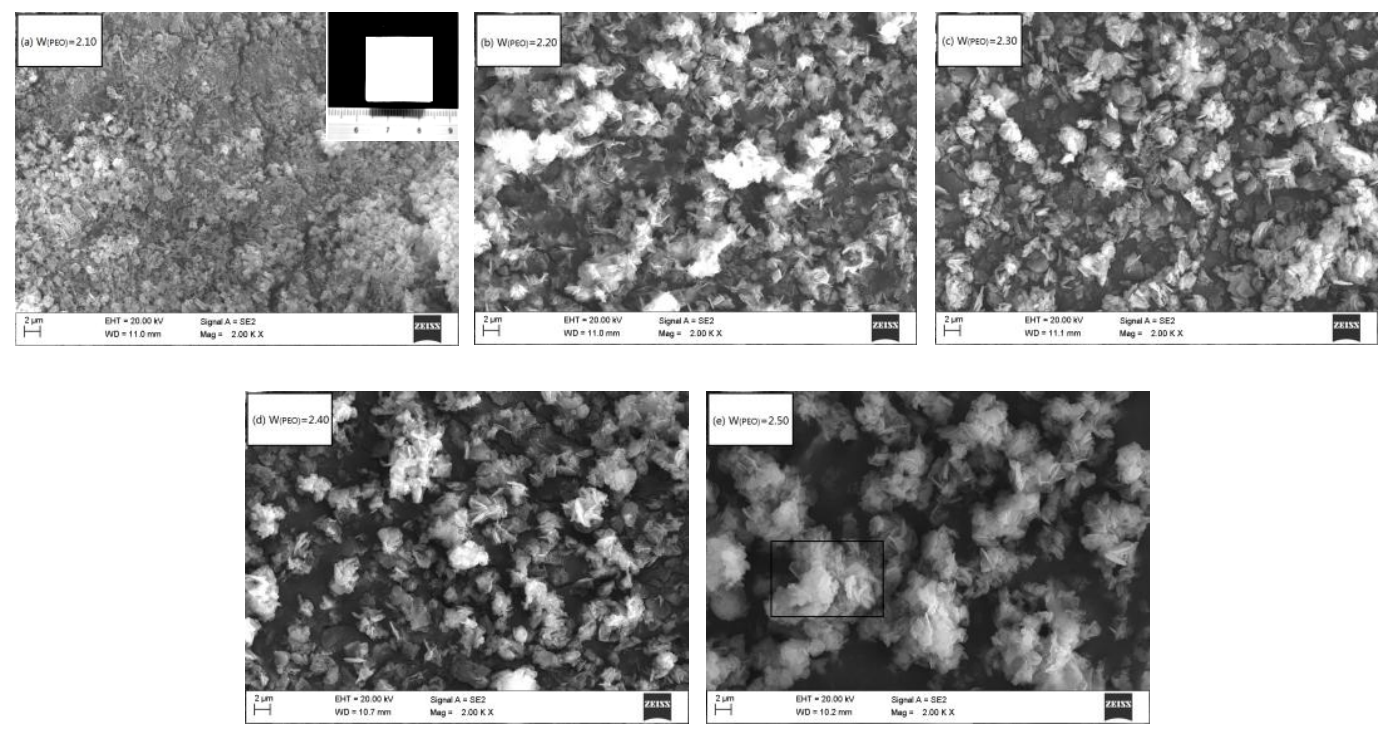

Fig. 2 Sectional microstructure of dried specimens 

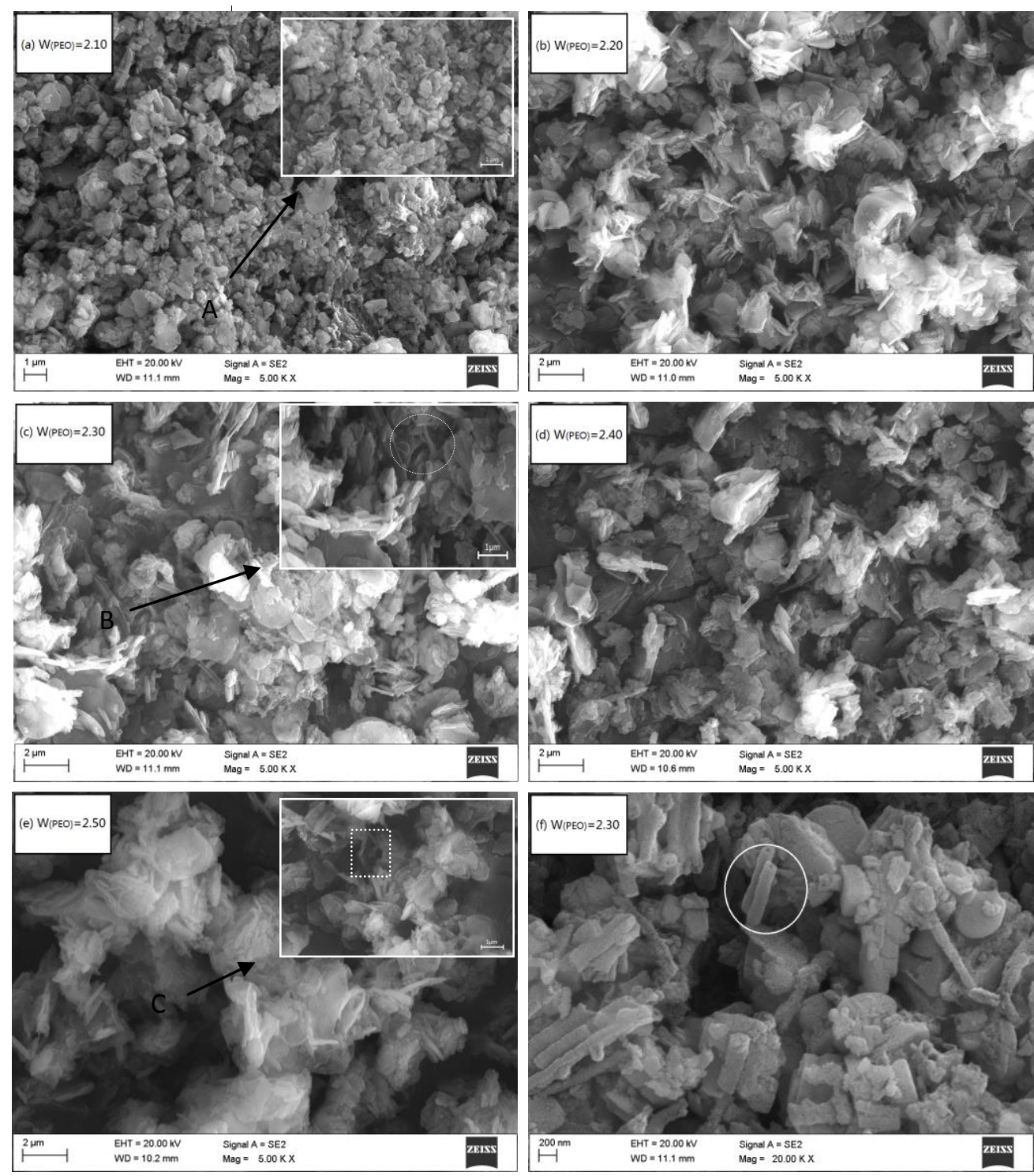

Fig. 3 Fracture scanning electron micrograph of $\mathrm{MgO}$ xerogel

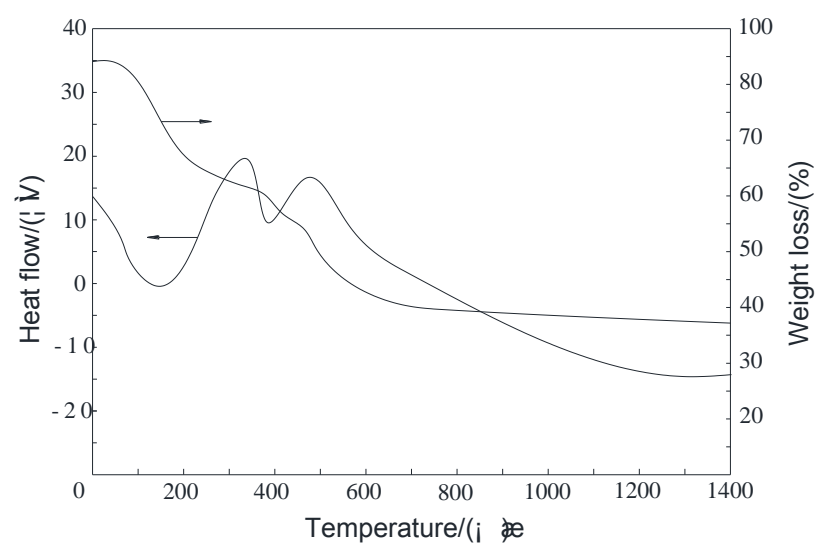

Fig. 4 Heat flow and weight loss curves: Specimen 3 


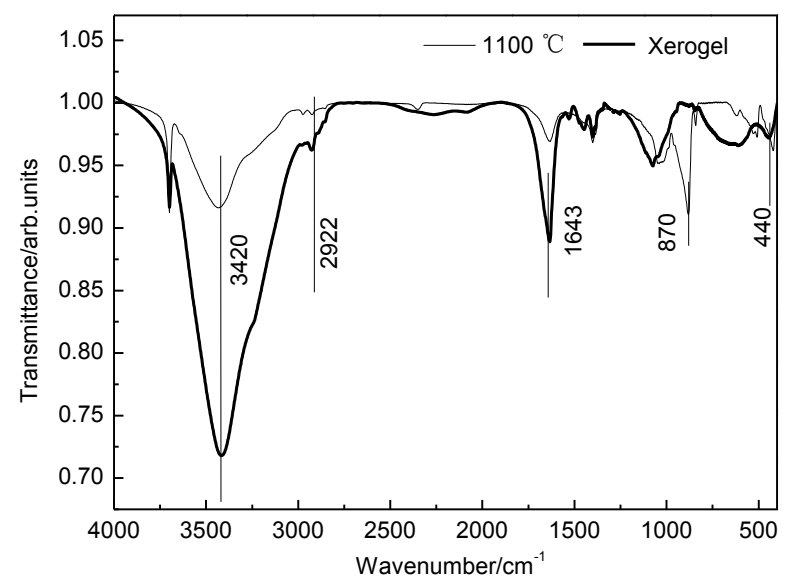

Fig. 5 FT-IR spectroscopy spectra for Specimen 3 (dried xerogel and sintered at $1100{ }^{\circ} \mathrm{C}$ )

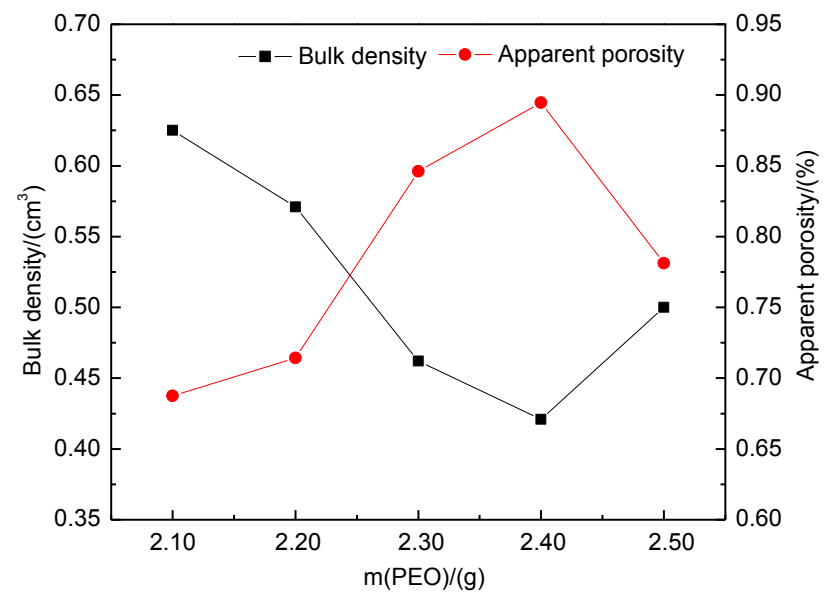

Fig. 6 Effect of PEO addition on bulk density and apparent porosity of the specimens after sintering 


\section{TABLE CAPTIONS}

Table 1 Ratio of raw materials

\begin{tabular}{ccccccc}
\hline Sample & $m\left(\mathrm{MgCl}_{2} \cdot 6 \mathrm{H}_{2} \mathrm{O}\right) / \mathrm{g}$ & $\begin{array}{c}V\left(\mathrm{H}_{2} \mathrm{O}\right) / \\
\mathrm{mL}\end{array}$ & $\begin{array}{c}V(\mathrm{EtOH}) / \\
\mathrm{mL}\end{array}$ & $\begin{array}{c}V(\mathrm{PO}) / \\
\mathrm{mL}\end{array}$ & $m(\mathrm{PEO}) / \mathrm{g}$ & $\begin{array}{c}V(\mathrm{TEOS}) / \\
\mathrm{mL}\end{array}$ \\
\hline 1 & 20 & 20 & 24 & 20 & 2.10 & 6.0 \\
2 & 20 & 20 & 24 & 20 & 2.20 & 6.0 \\
3 & 20 & 20 & 24 & 20 & 2.30 & 6.0 \\
4 & 20 & 20 & 24 & 20 & 2.40 & 6.0 \\
5 & 20 & 20 & 24 & 20 & 2.50 & 6.0 \\
\hline m- Mass; $V$ - Volume & & & & &
\end{tabular}

$m$-Mass; $V$ - Volume 